\title{
Comparison of Identification Methods of a Time-varying Insulin Sensitivity Parameter in a Simulation Model of Glucose Metabolism in the Critically Ill *
}

\author{
Ulrike Pielmeier* Steen Andreassen* Birgitte S. Nielsen** \\ Christopher E. Hann ${ }^{* * *}$ J. Geoffrey Chase ${ }^{* * *}$ \\ Pernille Haure** \\ * Center for Model-based Medical Decision Support, Aalborg University, \\ Fredrik-Bajers-Vej 7, 9220 Aalborg, Denmark (e-mail: \\ upiel@hst.aau.dk) \\ ** Neuro- and Trauma Intensive Care Unit, Aalborg Hospital, \\ Denmark \\ *** Department of Mechanical Engineering, Centre for \\ Bio-Engineering, University of Canterbury, Christchurch, NZ
}

\begin{abstract}
Models of glucose metabolism can help to simulate and predict the blood glucose response in hyperglycaemic, critically ill patients. Model prediction performance depends on a sufficiently accurate estimation of the patient's time-varying insulin sensitivity. The work presents three least squares approaches, the integral method and a Bayesian method that have been compared by prediction accuracy on an absolute and on a relative scale. Clinical data yields 1491 blood glucose predictions based on 10 critically ill patients were processed. The Bayesian approach proved to be best with small errors (9.7\% absolute percent error, 14.7 root mean square of logarithmic error for prediction times $\leq 2 \mathrm{~h}$ ), and fewer and smaller outliers compared to the other methods. Computationally, the Bayesian method took 1.5 times longer per prediction compared to the fastest method. It can be concluded that a Bayesian parameter estimation gives safe and effective results for the insulin sensitivity estimation for this model.
\end{abstract}

Keywords: insulin sensitivity, parameter identification methods, hyperglycaemia, critical care, metabolic modelling, glucose control.

\section{INTRODUCTION}

Hyperglycaemia is a frequent complication in the treatment of critically ill patients who present with or without previously diagnosed diabetes (McCowen et al., 2001). Since the first positive results from tight glycaemic control (TGC) (Van den Berghe et al., 2001; Krinsley, 2004) the means to achieve TGC have been subject of intense research (Meijering et al., 2006). In particular, it has been shown that intensive insulin therapy (IIT) can increase hypoglycaemia, which necessitates close monitoring IIT patients, and increases the clinical burden (Cryer, 2006; Devos and Preiser, 2007).

A number of model-based algorithms have been developed to simulate the blood glucose response to exogenous insulin treatment and nutrition (Wong et al., 2006; Plank et al., 2006). Virtual trials (Chase et al., 2008; Hovorka et al., 2008) allow estimating a model's capability to predict the longer term ( $>2$ hours) glycaemic response to intervention since accurate, longer-term predictions reduce the need for frequent blood glucose monitoring and thus reduce

\footnotetext{
^ This work was partially supported by a grant awarded by the Programme Commission on Nanoscience, Biotechnology and IT under the Danish Council for Strategic Research.
}

the clinical burden. Model-based simulations of blood glucose depend on the identification of model parameters to capture inter-subject variability as well as time-varying patient-specific dynamics. The identification method may be of importance for model predictive accuracy and thus, influence model performance in virtual trials.

In the present work, we look at five different methods to identify the time-varying patient-specific insulin sensitivity and evaluate the method impact on model-based prediction accuracy in shorter $(\leq 2$ hours) and longer-term ( $>2$ hours) predictions. More specifically, the Glucosafe model (Pielmeier et al., 2009) is used for with three different least squares methods, the integral method (Hann et al., 2005), and a Bayesian approach to identify insulin sensitivity. Methods are compared based on prediction accuracy and computational time.

\section{METHODS}

The Glucosafe model (Pielmeier et al., 2009) has been developed for blood glucose regulation in the critically ill. It is a composite metabolic model comprising four sub models: a two-compartment model of insulin kinetics, a model of saturation of insulin action at high insulin concentrations, a model of glucose absorption in the gut, 
and a blood glucose balance model. Insulin sensitivity is the patient-specific, time-varying parameter that needs to be identified for model prediction. In this model structure, the insulin sensitivity is a dimensionless factor $s \geq 0$ that links insulin effect and glucose levels. Reduced insulin sensitivity is thus modeled as scaling down the maximum effect of insulin on glucose uptake.

\subsection{Simulation conditions}

Simulations were run for each measurement $m_{i}$ in discrete steps of one minute, beginning with a run-in period $R$ followed by a parameter estimation period $E$ and concluded by a prediction period $P$ such that the duration $\Delta$ of the $i$-th simulation $S$ is:

$$
\Delta S_{i}=\Delta R_{i}+\Delta E_{i}+\Delta P_{i} .
$$

$P$ contained a set of measurements $\left\{m_{n+1}, . . m_{j}, . . m_{k}\right\}$. The prediction duration $\Delta P$ was 10 hours. $E$ contained a set of measurements $\left\{m_{1}, . . m_{i}, . . m_{n}\right\}$ and was defined to end at the time $\tau_{n}$ of the last measurement $m_{n}$. The duration $\Delta E$ varied with the identification method and the frequency of blood glucose measurements. The insulin sensitivity $s$ was estimated over $E$ and assumed to be constant over $S$.

$R$ preceded the parameter estimation period by at least 3 hours, and up to 24 hours if glycaemic data was available. I, Q, N, G are state variables and the run-in period lets them eliminate initial transients from the initial values defined:

$I$ : plasma insulin concentration

$=30 \mathrm{mU} / \mathrm{L}$

$Q$ : peripheral insulin concentration

$$
=18 \mathrm{mU} / \mathrm{L}
$$

$N$ : gut content

$=1 \mathrm{mmol}$ per $\mathrm{kg}$ bodymass

$G$ : simulated blood glucose

$=$ value of first measurement in $S$ in $\mathrm{mmol} / \mathrm{L}$.

All other variables were calculated as described in Pielmeier et al. (2009) and the endogenous insulin production was set to 35 units per day except patients with type 1 diabetes where the production rate was set to zero.

The value of $s$ was identified by fitting $G$ to the measured blood glucose concentrations $\left\{m_{1}, . ., m_{n}\right\}$ in $E$. The search space for $s$ was limited to values in $\{0 . .1\}$, where very low values indicate a high insulin resistance and 1 indicates a patient with normal insulin sensitivity. Five objective functions to identify $s$ were investigated (method acronyms in bold face). The global minimum of the argument of each method was searched over the search space of $s$, stopping when $s \pm 0.001$ yielded no further improvement.

\subsection{Parameter identification methods}

The first approach was the " 12 hour" least squares identification method used in Pielmeier et al. (2009) where the value of $s$ that minimizes the sum of the squares of residuals was found $(\mathbf{1 2 h})$ :

$$
\max _{s} \sum_{i=1}^{n}\left(\ln m_{i}-\ln G_{i}(s)\right)^{2}, \Delta E=12 \mathrm{~h}
$$

where $m_{i}$ is the $i$-th of $n$ measurements in $E$ and $G_{i}$ denotes the model-predicted blood glucose concentration of the $i$-th measurement. Residuals were calculated on a logarithmic scale because blood glucose measurements are approximately log-normally distributed as shown by Chase et al. (2008) and Pielmeier et al. (2009).

The second and third approach were both least squares methods derived from the $12 \mathrm{~h}$ method, but focused on recent changes in a patient's glycaemic state. For the second method, only the last two measured blood glucose concentrations were fitted $(\mathbf{2} \mathbf{p})$ :

$$
\max _{s} \sum_{i=n-1}^{n}\left(\ln m_{i}-\ln G_{i}(s)\right)^{2}, E=\left\{m_{n-1}, m_{n}\right\}
$$

where $\Delta E$ depends on the time between the last two measurements.

The third approach focused the most recent glycaemic state and $s$ was identified by fitting only to the last blood glucose measurement $m_{n}$, so $\Delta E=0$, and the objective function thus being $(\mathbf{1 p})$ :

$$
\max _{s} \sum_{i=1}^{n}\left(\ln m_{i}-\ln G_{i}(s)\right)^{2}, E=\left\{m_{n}\right\}
$$

The integral method of Hann et al. (2005) starts the simulation at time $\tau_{n-1}$, i.e. with $G_{n-1}=m_{n-1}$ as a limiting condition. Then they require that the simulation has the value $m_{n}$ at $\tau_{n}$, i.e. that $G_{n}(s)=m_{n}$ or (integ):

$$
\int_{\tau_{n-1}}^{\tau_{n}} \dot{G}(t \mid s) d t=m_{n}-m_{n-1},
$$

where $\dot{G}(t \mid s)$ is the derivative of the simulated blood glucose. As Hann et al. (2005) have shown, an explicit solution for $s$ can be found. The option to solve for $s$ explicitly was abandoned here in favor of the computationally more expensive approach of numerically searching for the value of $s$ that satisfies Equation 5 .

A Bayesian method was the final investigated approach. The general idea is to combine the empirical information contained in a set of samples $\left\{m_{1}, . ., m_{n}\right\}$ with the a priori knowledge about $s$ to obtain the a posteriori density function. In agreement with the previously mentioned lognormal distribution of blood glucose we define

$$
m_{i}^{*}=\ln m_{i}
$$

and

$$
\bar{m}_{i}^{*}=\left\{m_{1}^{*}, . . m_{n}^{*}\right\}
$$

If we assume that

$$
P\left(m_{i}^{*}\right)=N\left(\ln G_{i}, \sigma_{i}^{2}\right)
$$

then $s$ can be found by maximizing the a posteriori probability of $s$ given $m_{i}$ using Bayes' theorem:

$$
\max _{s} P\left(s \mid \bar{m}_{i}^{*}\right)=\frac{P\left(\bar{m}_{i}^{*} \mid s\right) P(s)}{P\left(\bar{m}_{i}^{*}\right)}=P\left(\bar{m}_{i}^{*} \mid s\right) P(s)
$$


because $P\left(\bar{m}_{i}^{*}\right)$ is a factor, independent of $s$, which does not change the maximization operation. Given that

$$
P\left(\bar{m}_{i} \mid s\right) \cong \prod_{i} P\left(m_{i} \mid s\right)
$$

and $P\left(m_{i}^{*} \mid s\right)$ being a normal density yields

$$
\begin{aligned}
& P\left(m_{i}^{*} \mid s\right)=\frac{1}{\sqrt{2 \pi} \sigma} \exp \left[-\frac{1}{2} \frac{\left(m_{i}^{*}-\ln G_{i}(s)\right)^{2}}{\sigma_{i}^{2}}\right] \Rightarrow \\
& P\left(m_{i}^{*} \mid s\right)=\prod_{i} \frac{1}{\sqrt{2 \pi} \sigma} \exp \left[-\frac{1}{2} \frac{\left(m_{i}^{*}-\ln G_{i}(s)\right)^{2}}{\sigma_{i}^{2}}\right]
\end{aligned}
$$

Further we assume that $s$ is also log-normally distributed:

$$
\begin{aligned}
P(\ln s) & \sim N\left(\ln \mu_{s}, \sigma_{s}^{2}\right) \\
& =\frac{1}{\sqrt{2 \pi} \sigma} \exp \left[-\frac{1}{2} \frac{\left(\ln s-\ln \mu_{s}\right)^{2}}{\sigma_{s}^{2}}\right] .
\end{aligned}
$$

Inserting Equations 11 and 12 into 9 yields

$$
\begin{aligned}
\max _{s} P\left(s \mid \bar{m}_{i}^{*}\right) & =\prod_{i} \frac{1}{\sqrt{2 \pi} \sigma_{i}} \exp \left[-\frac{1}{2} \frac{\left(m_{i}^{*}-\ln G_{i}(s)\right)^{2}}{\sigma_{i}^{2}}\right] \\
& \times \frac{1}{\sqrt{2 \pi} \sigma_{s}} \exp \left[-\frac{1}{2} \frac{\left(\ln s-\ln \mu_{s}\right)^{2}}{\sigma_{s}^{2}}\right]
\end{aligned}
$$

Removing constants gives:

$$
\begin{aligned}
\max _{s} P\left(s \mid \bar{m}_{i}^{*}\right) & =\prod_{i} \exp \left[-\frac{1}{2} \frac{\left(m_{i}^{*}-\ln G_{i}(s)\right)^{2}}{\sigma_{i}^{2}}\right] \\
& \times \exp \left[-\frac{1}{2} \frac{\left(\ln s-\ln \mu_{s}\right)^{2}}{\sigma_{s}^{2}}\right]
\end{aligned}
$$

Next we note that:

$$
\begin{aligned}
\max _{s} P\left(s \mid \bar{m}_{i}^{*}\right) & =\max _{s} \ln P\left(s \mid \bar{m}_{i}^{*}\right) \\
& =\left[-\frac{1}{2} \frac{\left(m_{i}-\ln G_{i}(s)\right)^{2}}{\sigma_{i}^{2}}\right] \\
& \times-\frac{1}{2} \frac{\left(\ln s-\ln \mu_{s}\right)^{2}}{\sigma_{s}^{2}} .
\end{aligned}
$$

Removing constants and changing sign yields (Bayes):

$$
\begin{aligned}
\max _{s} P\left(s \mid \bar{m}_{i}^{*}\right) & =\min _{s} \sum_{i=1}^{n} \frac{\left(\ln m_{i}-\ln G_{i}(s)\right)^{2}}{\sigma_{i}^{2}} \\
& +\frac{\left(\ln s-\ln \mu_{s}\right)^{2}}{\sigma_{s}^{2}}
\end{aligned}
$$

Choice of a priori parameters: We identified $\mu_{s}=0.257$ and $\sigma_{s}=0.091$ previously with these data (see subsection "Patient data" below) using the $12 \mathrm{~h}$ method presented earlier, yielding the probability density function $P_{1}(s)$ shown in Figure 1. However, this function is very narrow and reflects the small range of insulin sensitivities in a homogenously ill group of patients. Thus, for the Bayesian approach in this study, we propose to use function $P_{2}(s)$ instead as a priori probability function where $\mu_{s}=0.33$

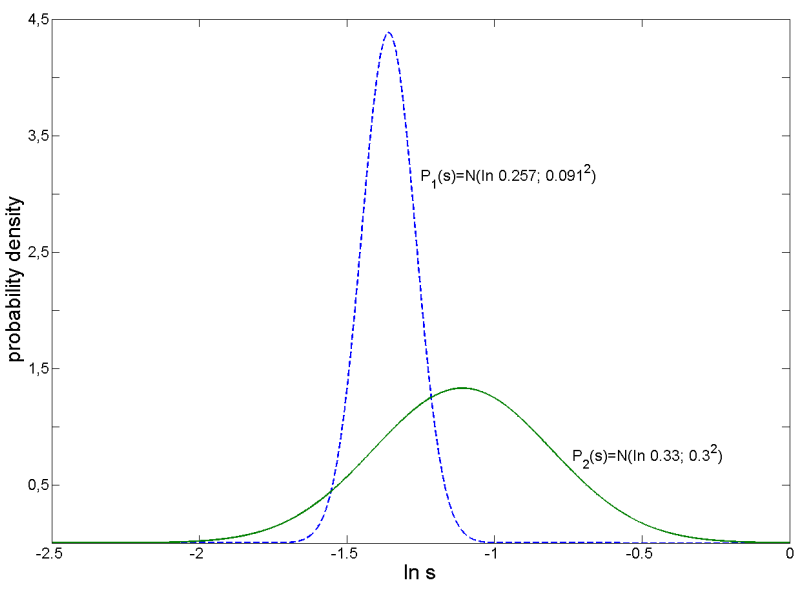

Fig. 1. Probability density functions for the natural logarithm of $s: P_{1}(s)$ with $\mu_{s}=0.257$ and $\sigma_{s}=0.091$ (Pielmeier et al., 2009); $P_{2}(s)$ the chosen a priori distribution with $\mu_{s}=0.33$ and $\sigma_{s}=0.3$.

Table 1. Method specifics and constraints

\begin{tabular}{llll} 
method & $\Delta E$ & $\begin{array}{l}\text { fitted } \\
\text { measurements }\end{array}$ & constraints \\
\hline $12 \mathrm{~h}$ & 12 hours & $m_{1}, . ., m_{n}$ & \\
$2 \mathrm{p}$ & $\tau_{n}-\tau_{n-1}$ & $m_{n-1}, m_{n}$ & \\
$\mathrm{lp}$ & 0 & $m_{n}$ & \\
integ & $\tau_{n}-\tau_{n-1}$ & $m_{n}$ & $G_{n-1}(s)=m_{n-1}$ \\
Bayes & $\tau_{n}-\tau_{1}$ & $m_{1}, . ., m_{n}$ & $\mu_{s}=0.33 ; \sigma_{s}=0.3 ;$ \\
& & & $\sigma_{0}=0.02 \sqrt{2}$ \\
& & & $\sigma_{m}=0.007$ \\
\hline
\end{tabular}

and $\sigma_{s}=0.3$ were values chosen to make the method applicable to a glycaemically broader range of patients.

The value for $\sigma_{i}$ was calculated using:

$$
\sigma_{i}=\sqrt{\sigma_{0}^{2}+\sigma_{m}^{2}\left(\tau_{n}-\tau_{i}\right)}
$$

where $\sigma_{0}$ is an expression for the variance due to measurement error associated with the assay method and $\sigma_{m}$ is a factor that increases the variance for measurements that are "older" compared to $m_{n}$, thus reducing the weight of measurements in proportion to a receding time horizon. For this study, $\sigma_{0}$ has been set to $0.02 \sqrt{2}$ that is an estimate of variance of two consecutive blood samples using the ABL ${ }^{\mathrm{TM}} 700$ blood gas analyzer (Pielmeier et al., 2009). The value for $\sigma_{m}$ was found by repeated calculation of the root mean square (RMS) of logarithmic prediction error over prediction time for different values of $\sigma_{m}$. Visual inspection of RMS results yielded $\sigma_{m}=0.007$.

A summary over method specifics and constraints is given in Table 1. Figure 2 shows an example of a model simulation for each of the five methods.

Over the prediction interval $P$, the predicted blood glucose value $G_{j}$ and the measured value $m_{j}$ were recorded as well as the length of the forecasted time $t_{j} \leq 10 \mathrm{~h}$ (i.e. the prediction time). Prediction errors were calculated on a logarithmic scale using:

$$
e_{j}=\ln m_{j}-\ln G_{j}
$$

and as absolute percent error (APE) using: 


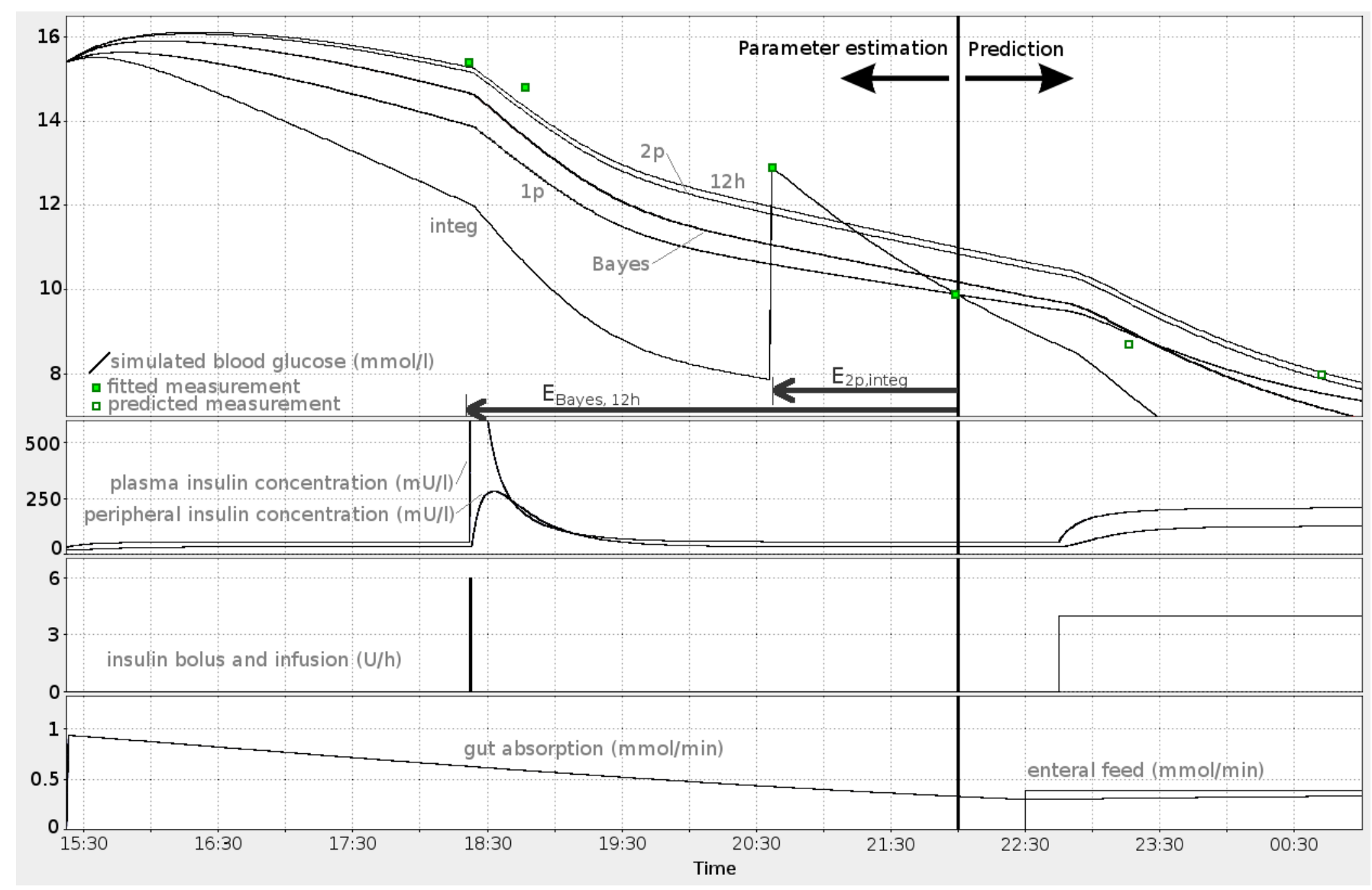

Fig. 2. Case scenario: The top panel shows the simulated blood glucose over time for each identification method. The identification interval $E$ stops at 22:00 at the time of the last measurement. The Bayes and the $12 \mathrm{~h}$ method fit four measurements over a longer $\Delta E_{\text {Bayes,12h }}$ compared to a shorter $\Delta E_{2 p \text {,integ }}$ for the $2 \mathrm{p}$ and the integ method that fit only the last two measurements. $\Delta E$ for the $1 \mathrm{p}$ method is zero. The right-hand side of the top panel shows two future measurements that will be predicted. In this case, Bayes and $1 \mathrm{p}$ method predict the first measurement more accurately while the $2 \mathrm{p}$ and $12 \mathrm{~h}$ method make a more accurate prediction on the second measurement. Panels 2,3 and 4 plot modelled insulin concentrations, exogenous insulin input and feed rate/gut absorption rate, respectively.

$$
A P E_{j}=\frac{\left|G_{j}-m_{j}\right|}{m_{j}} .
$$

The RMS of logarithmic prediction error and the median and interquartile range (IQR) of APE were calculated for 5 prediction time intervals of 2 hours length (number of predictions in parenthesis):

interval 1-2: $\quad$ predictions with $t \leq 2 \mathrm{~h}$ interval 3-4: predictions with $2 \mathrm{~h}<t \leq 4 \mathrm{~h}$ interval 5-6: $\quad$ predictions with $4 \mathrm{~h}<t \leq 6 \mathrm{~h}$ interval 7-8: $\quad$ predictions with $6 \mathrm{~h}<t \leq 8 \mathrm{~h}$ interval 9-10: predictions with $8 \mathrm{~h}<t \leq 10 \mathrm{~h}$

\subsection{Patient data}

Simulations were run with retrospective clinical data from 10 hyperglycaemic patients, admitted to the neuro- and trauma intensive care unit (ICU) at Aalborg hospital, Denmark, between 2005 and 2007. Glycaemic control had been carried out according to a local nurse-directed protocol that aimed for 5 to $8 \mathrm{mmol} / \mathrm{l}$ target blood glucose concentration. Insulin was given as intravenous infusions or as bolus injections. Blood samples were taken via an arterial

catheter and analyzed using a blood gas analyzer (ABL ${ }^{\mathrm{TM}} 700$, Radiometer A/S). Nutrition was given preferably by the enteral route and supplied with total parenteral nutrition if necessary. A detailed description of the patient characteristics and glycaemic control data can be found in (Pielmeier et al 2009). Ethical approval was given by the Ethical Committee for the Region of Northern Jutland.

\subsection{System requirements and performance measurement}

Simulations and performance measurements were processed on a laptop computer Dell Latitude D600 with Intel Pentium 1.6 GHz processor and installed Windows XP OS Version 2002. Program code was written in Java using JDK 1.6 and executed using Netbeans IDE version 6.0.1. Patient data were processed in batch mode, without multiple threads. Execution times are expressed on an absolute scale in seconds as arithmetic mean per simulation per patient and as multiples on a relative scale. SPSS software version 16.0 was used as statistical tool and MATLAB version 7.5 was used for curve fitting. 


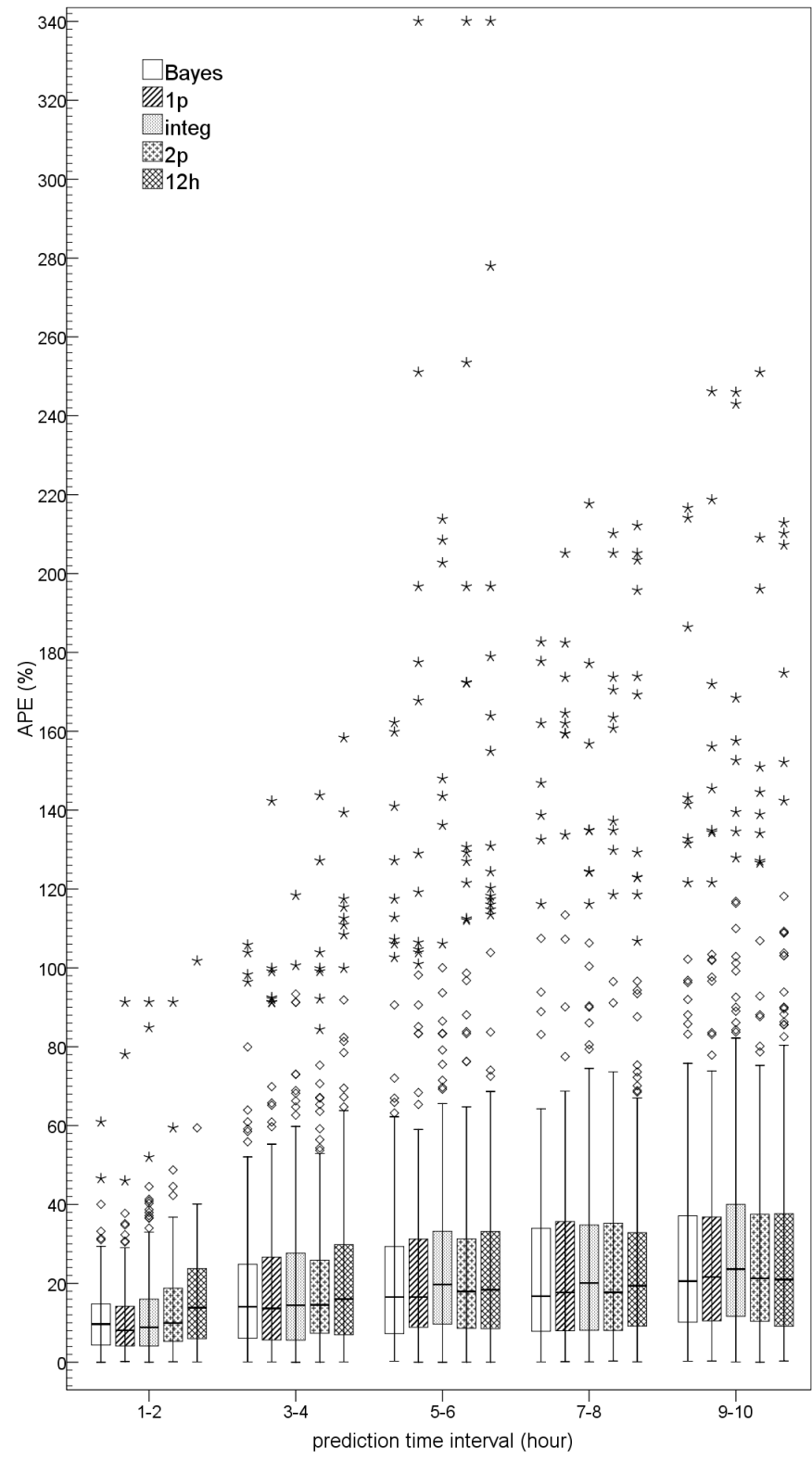

Fig. 3. Distribution of APE by method and prediction time interval. "Whiskers" extend to minimum and maximum values in the distribution not bigger than 1.5 times the interquartile range (IQR) as measured from the 75th percentile. Diamonds (asterisks) are outliers bigger than 1.5 (3) times the IQR as measured from the 75 th percentile.

\section{RESULTS}

Results of prediction errors are reported as box-andwhiskers plots and RMS diagram.

The box-and-whiskers plot in Figure 3 compares the methods by APE and prediction time interval. For longer prediction times, the error tends to increase for all methods. Median, 25th and 75th percentile are lowest for the 1point method and highest for the $12 \mathrm{~h}$ method for short prediction times $\leq 2 \mathrm{~h}$. At longer prediction times, medians, 25th and 75th percentile tend to be lowest for the Bayes method. Over all prediction times outliers were smaller for the Bayes method.

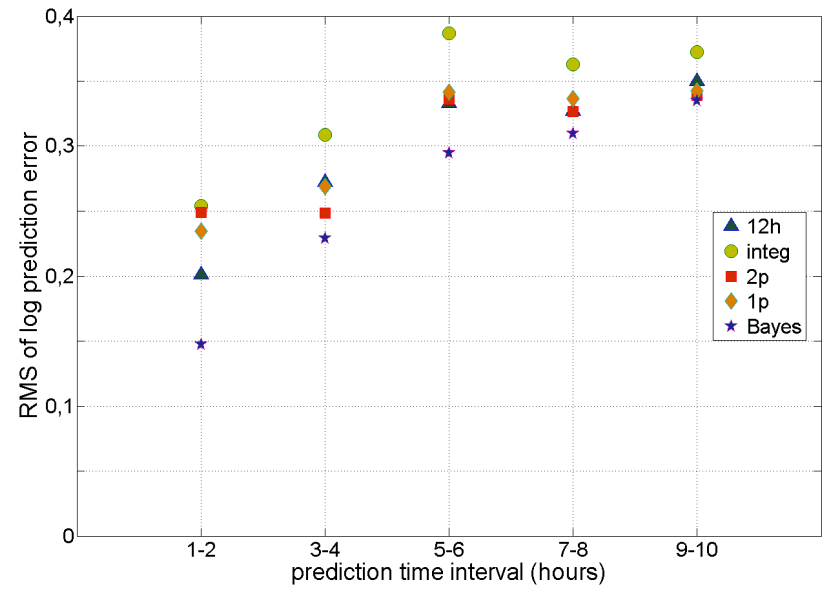

Fig. 4. Root mean square (RMS) of logarithmic prediction error for the five identification methods.

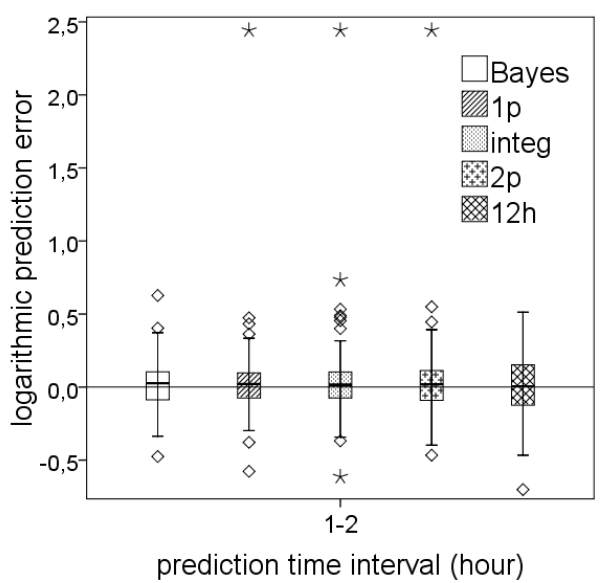

Fig. 5. Logarithmic prediction error $e_{j}=\ln m_{j}-\ln G_{j}$ for prediction times $\leq 2$ hours.

Figure 4 compares the methods by RMS of logarithmic prediction error and prediction time interval. It can be seen that the Bayes method gave the smallest errors over all prediction time intervals compared to the other methods. Figure 5 shows a detailed view over short-term predictions (1-2 hours forward). Outliers seen in Figure 5 mark a case where the identified insulin sensitivity by the $1 \mathrm{p}$ method, integ method and the $2 \mathrm{p}$ method was highly overestimated, yielding a largely underpredicted blood glucose. This example shows how single large errors affect the RMS of prediction error though the effect on the median is not distinct. Figures 3 and 4 also show a decreasing method effect on error outcome for increasing prediction time intervals: at prediction times $>9$ hours the APE and logarithmic prediction errors are very much the same for the different methods.

Computational times are listed as mean identification time per measurement in Table 2. On average, the 1p method was fastest per identification. The Bayes method took on average 1.50 times longer. 
Table 2. Computational times *

\begin{tabular}{lll} 
method & $\begin{array}{l}\text { absolute time } \\
\text { per measurement }(\mathrm{sec})\end{array}$ & $\begin{array}{l}\text { time per measurement } \\
\text { relative to 1point method }\end{array}$ \\
\hline $12 \mathrm{~h}$ & 0.50 & 1.39 \\
$2 \mathrm{p}$ & 0.40 & 1.11 \\
$\mathrm{lp}$ & 0.36 & 1.00 \\
integ & 0.43 & 1.19 \\
Bayes & 0.54 & 1.50 \\
\hline
\end{tabular}

* The integral method would have been computationally much faster had an explicit solution for $s$ been implemented.

\section{DISCUSSION}

The present work compared five methods for identification of the insulin sensitivity in a simulation model of glucose metabolism. The comparison was carried out from 1491 blood glucose predictions from retrospective glycaemic data of 10 critically ill patients and calculation of the accuracy of predictions on an absolute and a relative scale. Additionally, the computational time needed by each method was recorded and compared.

For short prediction times ( $<2$ hours) comparing absolute percent error, results showed a slight advantage of the $1 \mathrm{p}$ method that based the parameter estimation entirely on the last measured blood glucose concentration. However, the Bayes method gave fewer and smaller outliers for the same prediction time interval on an absolute scale, and outperformed the other methods firmly when compared by root mean square on a relative scale. The computational disadvantage was small since a prediction using the Bayes method required on average only 1.5 times longer than the fastest method (1p).

It can be concluded that the Bayesian approach is a reasonable and effective method to identify the insulin sensitivity for simulations using this model. Overall, prediction error is a function of sensor error (small and random) and evolution or changes in condition over the prediction time period, which can be quite large in critical care (Lin et al. (2008)). The Bayesian approach presented performs better for outliers, and thus overall, because it manages this variability with its a priori probability density functions, as seen in Figure 1, limiting or accounting for the range of possible changes and liklihood. In contrast, Lin et al. (2008) used this likelihood from their stochastic model to moderate the intervention. One interesting future avenue would be to replace $P_{2}(s)$ in Figure 1 with a data based and dynamic function of current insulin sensitivity based off the stochastic models like those of Lin et al. (2008).

\section{REFERENCES}

Chase, J.G., LeCompte, A., Shaw, G., Blakemore, A. Wong, J., Lin, J., and Hann, C.E. (2008). A Benchmark Data Set for Model-Based Glycemic Control in Critical Care. J Diabetes Sci Technol, 2(4), 584-594.

Cryer, P.E. (2006). Hypoglycaemia: the limiting factor in the glycaemic management of the critically ill? Diabetologia, 49, 1722-1725.

Devos, P., and Preiser, J.C. (2007). Current controversies around tight glucose control in critically ill patients. Curr Opin Clin Nutr Metab Care, 10, 206-209.
Hann, C.E., Chase, J.G., Lin, J., Lotz, T., Doran, C.V., Shaw, G.M. (2005). Integral-based parameter identification for long-term dynamic verification of a glucoseinsulin system model. Comp Meth Progr Biomed., 77(3), 259-270.

Hovorka, R., Chassin, L.J., Ellmerer, M., Plank, J., Wilinska, M.E. (2008). A simulation model of glucose regulation in the critically ill. Physiol.Meas., 29, 959978.

Krinsley, J.S. (2004). Effect of an intensive glucose management protocol on the mortality of critically ill adult patients. Mayo Clin Proc, 79, 992-1000.

Lin, J., Lee, D.S., Chase, J.G., Shaw, G.M., LeCompte, A., Lotz, T., Wong, X.W., Lonergan, T. and Hann, C.E. (2008). Stochastic modelling of insulin sensitivity and adaptive glycemic control for critical care Comp Meth Progr Biomed., 89(2), 141-52, ISSN: 0169-2607.

McCowen, K.C., Malhotra, A., and Bistrian, B.R. (2001). Stress-induced hyperglycemia. Crit Care Clin, 17(1), 107-124.

Meijering, S., Corstjens, A.M., Tulleken, J.E., Meertens, J.H.J.M., Zijlstra, J.G., and Ligtenberg, J.J.M. (2006). Towards a feasible algorithm for tight glycaemic control in critically ill patients: a systematic review of the literature. Crit Care, 10, R19 (doi:10.1186/cc3981).

Pielmeier, U., Andreassen, S., Nielsen, B.S., Chase, J.G., Haure, P. (2009). A model of insulin saturation and glucose balance for glycaemic control in ICU patients. In preparation.

Plank, J., Blaha, J., Cordingley, J., Wilinska, M.E., Chassin, L.J., Morgan, C., Squire, S., Haluzik, M., Kremen, J., Svacina, S., Toller, W., Plasnik, A., Ellmerer, M., Hovorka, R., and Pieber, T.R. (2006). Multicentric, randomized, controlled trial to evaluate blood glucose control by the model predictive control algorithm versus routine glucose management protocols in intensive care unit patients. Diabetes Care, 29(2), 271-276.

Van den Berghe, G., Wouters, P., Weekers, F., Verwaest, C., Bruyninckx, F., Schetz, M., Vlasselaers, D., Ferdinande, P., Lauwers, P., Bouillon, R. (2001). Intensive insulin treatment in critically ill patients. $N$ Engl $\mathrm{J} \mathrm{Med,}$ 345, $1359-1367$.

Wong, X.W., Singh-Lewett, I., Hollingsworth, L.J., Shaw, G.M., Hann, C.E., Lotz, T., Lin, J., Wong, O.S., and Chase, J.G. (2006). A novel model-based insulin and nutrition delivery controller for glycaemic regulation in critically ill patients. Diabetes Technol Ther, 8, 174190. 